\title{
Inconsistências no Processo de Recomendação de Objetos de Aprendizagem utilizando Teoria de Estilos de Aprendizagem
}

\author{
Ana C. Rodrigues ${ }^{1}$, Luciana Assis ${ }^{1}$, Alessandro Vivas ${ }^{1}$, \\ Cristiano Pitangui ${ }^{2}$, Cristiano M. Silva ${ }^{2}$ \\ ${ }^{1}$ Universidade Federal dos Vales Jequitinhonha e Mucuri (UFVJM) \\ CEP 39100-000 Diamantina - MG - Brasil \\ ${ }^{2}$ Universidade Federal de São João del-Rei (UFSJ) \\ CEP 36307-352 São João del-Rei - MG - Brasil \\ \{rodrigues.carolina, lpassis, alessandrovivas\}@ufvjm.edu.br \\ \{pitangui.cristiano, cristiano\}@ufsj.edu.br
}

\begin{abstract}
A recomendação de Objetos de Aprendizagem e detecção de Estilos de Aprendizagem tem atraído interesse de muitos pesquisadores. Este artigo descreve os resultados alcançados com a execução da Revisão Sistemática da Literatura, cujo objetivo é mapear os Estilos de Aprendizagem e Objetos de Aprendizagem. Os resultados apontaram inúmeras inconsistências na forma como os recursos educativos são indicados a determinados perfis de alunos, suscitando questionamentos acerca da efetividade dos Estilos de Aprendizagem no processo de recomendação de Objetos de Aprendizagem.
\end{abstract}

Resumo. The Recommendation of Learning Objects and Learning Styles detection has attract interest from many researchers. This article describes the results achieved with the implementation of the Systematic Review of Literature, whose objective is to map the Learning Styles and Learning Objects. The results pointed out inconsistencies numerous in the way in which the educational resources are indicated to certain profiles of students, raising questions about the effectiveness of the Learning Styles in the process of recommendation of Learning Objects.

\section{Introdução}

A Educação a Distância (EaD) é um tema explorado com magnitude pela literatura, várias abordagens são objetos de estudo, como por exemplo a adaptação do ensino. Um dos objetivos em adaptar o processo ensino-aprendizagem é fornecer ao aluno um ambiente que seja condizente com as suas preferências individuais de aprendizado. Os resultados provenientes das pesquisas realizadas por [Li et al. 2012] e [Carvalho 2015], demonstram progressos positivos no rendimento do aluno quando o conteúdo é apresentado em concordância com o seu perfil. Portanto, a adaptação do processo ensino/aprendizagem é uma área em potencial para promover a melhoria da oferta da Educação a Distância.

Dois importantes conceitos, o Estilo de Aprendizagem (EA) e Objeto de Aprendizagem (OA), interligados à adaptabilidade do ensino, são o foco de estudo deste trabalho. Segundo a definição de [Felder et al. 1988], o Estilo de Aprendizagem são as preferências 
individuais de aprendizado do aluno, definidas conforme o modo de percepção, processamento da informação e solução de problemas de cada indivíduo. Já o Objeto de Aprendizagem refere-se às ações instrucionais indicadas aos alunos, assim, são exemplos de OA, os recursos educativos: vídeos, imagens, palestras, jogos, dentre outros. De acordo com [Behar et al. 2007], o objeto de aprendizagem propicia a diversificação da apresentação do conteúdo aos alunos e enriquece o espaço pedagógico.

Diversas pesquisas envolvendo personalização de ensino associam EA e OA, ou seja, o conteúdo é apresentado ao aluno através de OA que vão de encontro com as preferências de aprendizado ditadas por seu EA. O intuito dos Sistemas de Tutoria Inteligentes é prover sistemas instrucionais que operam sob esta perspectiva. Sabemos que outros fatores são considerados para propiciar a adaptação do ensino, como por exemplo, o contexto do aluno e a tecnologia por ele utilizada. No entanto, o objetivo do presente trabalho é estudar a relação entre o OA e EA e apresentar um mapeamento de quais recursos educativos são indicados a determinados perfis de alunos. Assim, as perguntas que nortearam a pesquisa foram: quais os tipos de OA são adotados pelos Sistemas de Tutoria Inteligentes? O número de tipos de objetos de aprendizagem relacionado a cada EA é equipendente? Pode-se falar em correlação/mapeamento entre EA e OA?

A motivação em realizar esta pesquisa se deve ao crescimento expressivo e em curto prazo do número de trabalhos que abordam ambos os conceitos: EA e OA. Estes estão relacionados em abordagens diferentes. A primeira delas é a detecção do Estilo de Aprendizagem do aluno e posterior recomendação dos Objetos de Aprendizagem, como se percebe em [Wenger 2014], [Silva et al. 2014], [Peña et al. 2002]. A outra abordagem é a detecção do EA do aluno a partir da verificação dos OA acessados durante o seu itinerário pedagógico como em [Franzoni et al. 2008], [Kolekar et al. 2017].

Assim, o intuito é saber como emerge, nestes contextos, a relação entre o EA e o OA. Além disso, encontrar pressupostos que indiquem o motivo pelo qual esta temática é explorada com dinamismo pela comunidade acadêmica, a ponto de contemplar em um período temporal curto, diversos trabalhos com esta abordagem.

Por outro lado, também despontam publicações que refutam o uso dos EA. [Rohrer and Pashler 2012], [Curry 1990], [Coffield 2004], alegam confusão nas definições; fraqueza na confiabilidade e validade das medições; ineficiência da identificação de características relevantes sobre os aprendizes e as instruções e falta de estudos empíricos. Pretende-se com esta revisão obter subsídios que possam contrapor ou apoiar o mito do EA, uma vez que, o processo de personalização, em muitas pesquisas, é baseado no Estilo de Aprendizado do aluno.

Para alcançar este objetivo, adotamos como método a Revisão Sistemática da Literatura e, posteriormente, extrair resultados das publicações recuperadas associadas a um determinado assunto [Kitchenham 2004].

Outras revisões sistemáticas da literatura já foram realizadas envolvendo EA e OA. [Valaski et al. 2011] analisam os modelos de estilos de aprendizagem utilizados no âmbito da adaptação dos recursos de aprendizagem. Foram analisados trabalhos entre os anos de 2005 a 2011 e os resultados da pesquisa mostraram que os modelos mais utilizados, nesta ordem, foram: Felder e Silverman, Kolb, Vark e Keefes. Os dados obtidos na pesquisa não permitiram mapear os Objetos de Aprendizagem e Estilos de Aprendiza- 
gem. Os autores apresentam apenas as características gerais dos recursos educativos que pertencem a cada EA. O principal diferencial deste trabalho é a identificação dos OA's encontrados na literatura e apresentação do seu relacionamento com um EA.

Com uma perspectiva mais abrangente que o trabalho desenvolvido por [Valaski et al. 2011], a Revisão Sistemática da Literatura desenvolvida por [do Nascimento et al. 2017] identificou: os modelos de EA utilizados durante o processo de recomendação automática e personalizada de conteúdos; verificação dos padrões de metadados de OA adotados (LOM, SCORM); os AVA's e mecanismos de apoio aplicados no contexto da recomendação adaptativa de recursos de aprendizagem. Foram recuperados trabalhos entre os anos de 2002 e 2016. Os resultados apresentados indicaram que o modelo de Felder e Silverman predomina nos trabalhos analisados, assim como na pesquisa mencionada anteriormente. Os demais modelos de EA mencionados na literatura foram Kolb, Vark e Honey-Alonso.

O presente trabalho visa acrescentar às pesquisas existentes um estudo que apresente, principalmente, como os trabalhos recuperados na RSL associam os EA e tipos de OA, sendo este, o maior diferencial da pesquisa proposta em relação aquelas já publicadas.

\section{Metodologia}

A Revisão Sistemática da Literatura proposta por [Kitchenham 2004] contempla três etapas. A primeira delas é o planejamento, etapa em que se faz a identificação das questões de pesquisa e a elaboração do protocolo de revisão. Na segunda fase ocorre a seleção dos estudos, que é guiada pelo protocolo elaborado na primeira etapa. Por fim, a terceira etapa realiza a análise dos resultados e os mesmos são reportados.

Na etapa de planejamento, é necessário definir um protocolo de revisão que especifica os métodos que serão utilizados para conduzir a RSL [Kitchenham 2004]. Os elementos que compõem este protocolo são: objetivo e justificativa para a pesquisa, questões de pesquisa, estratégia utilizada para realizar a busca de estudos primários e critérios de seleção e exclusão de trabalhos. O primeiro elemento a ser elaborado é o objetivo para execução da RSL, assim procuramos investigar a relação entre os estilos de aprendizagem e os objetos de aprendizagem, durante o processo de recomendação automática e personalizada de conteúdos. Com isso, as seguintes questões de pesquisas foram delineadas:

[QP] É possível relacionar os tipos de Objetos de Aprendizagem aos Estilos de Aprendizagem do aluno considerando a forma como estes OAs são recomendados para cada EA na literatura?

Algumas questões específicas (QE), utilizadas para auxiliar a responder a questão principal, foram definidas:

QE[1] Os tipos de Objetos de Aprendizagem são recomendados de acordo com os estilos de aprendizagem dos alunos?

QE[2] Quais tipos de Objetos de Aprendizagem são mais indicados a determinados estilos de aprendizagem? É possível falar em correlação entre EA e OA?

Para recuperação dos artigos foram utilizadas as bases de dados ACM Digital Library, IEEE Xplore Digital Library, Science Direct, Scopus e Google Acadêmico. 
O período temporal considerado foi entre 2002 a 2018. Os termos selecionados para construção da string de pesquisa foram: (Recomendação OR Personalização OR Indicação OR Detecção OR Mapeamento) AND (Objetos de Aprendizagem OR Recursos Educativos OR Objetos Educacionais) AND (Estilos de Aprendizagem OR Perfil do Aluno OR Preferências de Aprendizagem OR Estilso Cognitivos OR Estilos de Aprendizado). Vale ressaltar que no decorrer da pesquisa a string foi calibrada para propiciar o retorno efetivo de trabalhos que falam sobre a temática em estudo, como indicado por [Neiva and da Silva 2016].

Após definir a estratégia de busca, é necessário analisar o processo de eliminação dos trabalhos. Neste artigo, o processo foi composto por três etapas, a primeira eliminação baseou-se na leitura conjunta do título, resumo e conclusão, visto que geralmente o título não fornece detalhes suficientes para a avaliação do material. Considerando que a fase de eliminação dos trabalhos envolve a subjetividade do pesquisador, como apontando por [do Nascimento et al. 2017], algumas estratégias para guiar o processo foram a definição de critérios de exclusão e de qualidade. Uma medida para verificar se o artigo atendia ou não ao objetivo da pesquisa foi a definição de 06 critérios de qualidade ${ }^{1}$.

Após concluir a primeira etapa da revisão, o próximo passo é a Condução. Assim, os artigos mantidos na primeira fase (título e resumo) passaram pela eliminação por leitura diagonal e posteriormente pela leitura completa. A Tabela 2 indica a quantidade de trabalhos eliminados em cada fase do processo de exclusão. Foram recuperados um total de 110 trabalhos na base ACM Digital Library, 63 com a busca por IEEE Xplore, 24 publicações oriundas da base Science Direct, 64 trabalhos obtidos com a pesquisa no Google Acadêmico e 37 na Scopus, totalizando 298 trabalhos recuperados. Uma precaução a ser tomada a fim de evitar erros na execução da SRL é tomar por definição que, em caso de dúvida se um artigo deveria ou não ser eliminado, o mesmo deveria ser mantido para análise na etapa seguinte. Assim, 40 trabalhos foram excluídos na primeira fase (Título e Resumo). A Figura 1 representa separadamente a exclusão nas respectivas bases de dados. A eliminação tornou-se mais expressiva na segunda fase (Leitura Diagonal), quando foram excluídos 119 trabalhos e, por fim, 114 trabalhos com a Leitura Completa, restando 37 artigos para consolidação dos resultados.

Os artigos mantidos na Etapa 3 ( leitura completa) foram revisados com o objetivo de extrair as respostas que condiziam com as questões de pesquisa. A identificação dos OA e a sua relação com os EA era uma delas. Devido ao fato das publicações recuperadas estarem em três idiomas, foi necessário analisar a natureza de cada objeto de aprendizagem, uma vez que diferentes palavras eram utilizadas com sentidos e objetivos distintos ou não. O objeto exercício, por exemplo, era empregado na modalidade de autoavaliação, discursivos e múltipla escolha. Quando termos diferentes eram utilizados para caracterizar a mesma natureza de atividade, os mesmos eram mesclados e categorizados na mesma classe. Por sua vez, se os OA apresentassem objetivos distintos, categorias diferentes eram criadas.

Posteriormente, realizou-se a associação destes objetos com os perfis dos alunos. Para isso, os artigos foram analisados cuidadosamente. O relacionamento foi criado conforme a indicação dos OA para os EA. Em cada artigo foram identificados os tipos de OA

\footnotetext{
${ }^{1}$ Esses métodos empregados encontram-se disponíveis em:

https://drive.google.com/file/d/1wdZIGIsPqyj9FDbDPHxAm8B-CUY8cswr/view?usp=sharing
} 


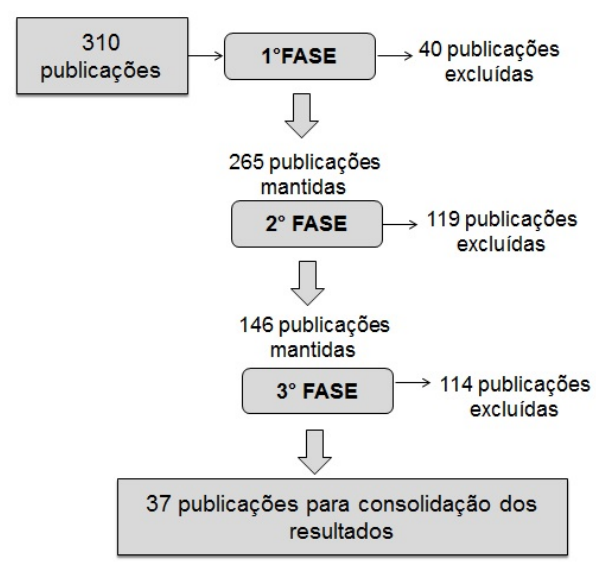

Figura 1. Relação dos filtros realizados e correspondentes eliminações

e contabilizada a ocorrência nos respectivos EA.

\section{Resultados e Discussões}

Os artigos selecionados para extração de resultados reuniram informações úteis que estavam relacionadas às questões de pesquisa do trabalho. Os 37 trabalhos recuperados abordavam o modelo de EA de Felder e Silverman. Esta predominância do modelo de Felder e Silverman é justificada por [Valaski et al. 2011], [Graf et al. 2009] e [Dorça et al. 2013] pela disponibilidade e amplo conjunto de informações que versam sobre as dimensões do modelo e suas relações com os recursos de aprendizagem adaptados, também, por utilizar o conceito de dimensões, o torna claro e completo de informações.

O modelo de Felder e Silverman organiza as informações conforme estágios de aprendizagem. O processamento da informação refere-se ao modo como os estudantes agem sobre o conteúdo, englobando a dicotomia de estilos Ativo e Reflexivo. Os alunos considerados como Ativos preferem aprender de forma ativa, ou seja, tendem a aprender agindo sob o conteúdo. Por sua vez, alunos com perfil Reflexivo optam por processar conteúdos teóricos, que favorecem o exercício do pensamento para refletir as informações repassadas [Felder et al. 1988].

A dimensão Entrada do modelo integra o EA Visual e Verbal, e está relacionados ao modo como o aluno deseja receber as informações, seja por instrumentos visuais representativos (Estilo Visual) ou por meio da apresentação do conteúdo via formato de texto ou áudio (Estilo Verbal). A dimensão Percepção está relacionada ao modo como o estudante percebe o conteúdo, e inclui os estilos Sensitivo e Intuitivo. O estilo Intuitivo configura os estudantes que preferem conteúdos que induzem a abstração, reflexão e imaginação. Por outro lado, o estilo Sensitivo caracteriza os alunos que preferem associar as informações processadas com o mundo real por meio de testes práticos [Dorça et al. 2011].

Por fim, a dimensão Organização da informação indica o modo como o aluno prefere receber a evolução e sequenciamento das informações apresentadas. O estilo Sequencial caracteriza o estudante que opta pelo aprendizado adquirido passo a passo.

Assim, filtramos os trabalhos que abordavam o modelo de EA de Felder e Silverman para analisar as informações que iam de encontro com a [QP] É possível relacionar 
os tipos de objetos de aprendizagem que são indicados de acordo com o estilo de aprendizagem do aluno?

Foi encontrado um número expressivo de publicações que abordam este tema. É colocada em discussão a adaptação de conteúdos de acordo com o estilo de aprendizagem do aluno e a detecção do EA de acordo com OA acessados. Dessa maneira, fica evidente a associação entre o EA e o OA. Comprovamos e apresentamos esta correlação na Figura 2, que mostra a relação dos OA catalogados, respondendo a primeira pergunta de pesquisa: . Os valores apresentados na figura indicam a porcentagem de OA catalogados em cada EA. A figura foi esquematizada conforme a contagem dos OA em cada EA em relação ao total de OA recuperados com a RSL.

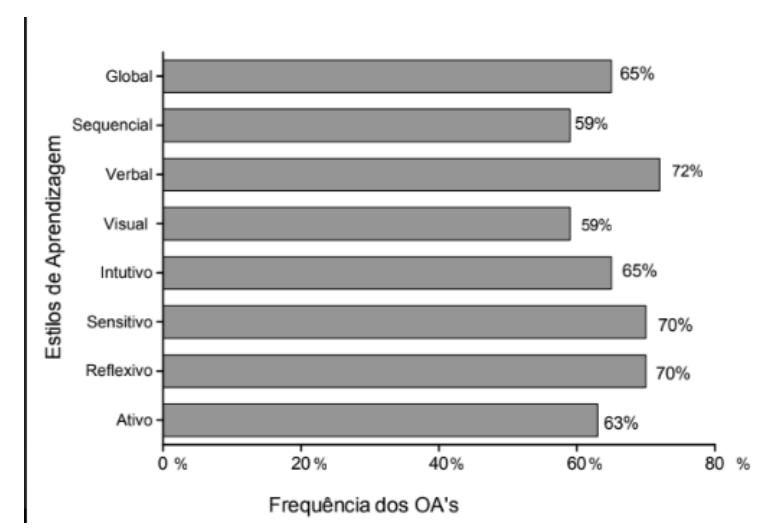

Figura 2. Ocorrência dos Objetos de Aprendizagem presentes em cada Estilo de Aprendizagem.

Das publicações recuperadas, encontrou-se um número expressivo de pesquisas que discutem sobre a adaptação de conteúdos de acordo com o perfil do aluno. Diferentes abordagens e métodos, além do foco de pesquisa especificado neste trabalho, são discutidos, como o enfoque para aprendizagem inclusiva (pessoas com deficiência) e a recomendação dos objetos de aprendizagem de acordo com a tecnologia utilizada pelo aluno. Assim, é perceptível que a temática em questão é uma área de pesquisa de grande interesse na comunidade acadêmica. Os autores concordam que a adaptabilidade do ensino é essencial para garantir um aprendizado eficaz e personalizado, além de potencializar as chances de permanência do aluno no curso [Zaina 2010],[Franzoni et al. 2008]

Os resultados iniciais obtidos com a análise dos artigos permitiram responder a questão de pesquisa principal que norteia os questionamentos específicos e fornece subsídios aos mesmos. O próximo passo era encontrar pressupostos que pudessem argumentar a primeira questão de pesquisa específica:QE[1] Os objetos de aprendizagem são recomendados de acordo com os estilos de aprendizagem dos alunos?

A revisão permitiu identificar 46 Objetos de Aprendizagem ${ }^{2}$ utilizados nas publicações recuperadas, respondendo também à primeira pergunta de pesquisa: quais os tipos de OA são adotados pelos Sistemas de Tutoria Inteligentes?

\footnotetext{
${ }^{2}$ Lista de tipos de OA recuperados na RSL está disponível em: https://drive.google.com/file/d/1bxu2nhVRvjhV1PV2dVJQQH8vdclx911/view?usp=sharing
} 
Todos os EA estavam associados a tipos de OA conforme características de cada dimensão. Portanto, a associação entre os dois elementos não é realizada de forma aleatória, mas ao contrário: os recursos educativos são indicados conforme preferência de aprendizagem do aluno. Além disso, o gráfico apresentado na Figura 2 mostra que não há diferenças significativas entre o número de objetos recuperados para cada estilo, bem como nenhum estilo se destaca na quantidade de objetos de aprendizagem associados, o que responde à segunda pergunta de pesquisa: o número de tipos de objetos de aprendizagem relacionado a cada EA é equipendente?

Em seguida, o próximo questionamento era QE[2] Quais objetos de aprendizagem são mais indicados a determinados estilos de aprendizagem do modelo de Felder e Silverman? Sabendo-se que era possível mapear os OA aos respectivos EA, e que a associação entre os dois itens é feita com o objetivo de ofertar ao aluno o conteúdo de forma efetiva levando em consideração o seu EA, o mapeamento foi construído gerando os gráficos relativos a cada perfil das dimensões. A Tabela 1 demonstra a quantidade de Objetos de Aprendizagem recuperados em cada Estilo de Aprendizagem ${ }^{3}$.

\begin{tabular}{|c|c|}
\hline \multicolumn{2}{|c|}{ Estilos de Aprendizagem } \\
\hline Ativo: 29 & Reflexivo: 32 \\
\hline Sensitivo: 32 & Intuitivo: 30 \\
\hline Visual: 25 & Verbal: 33 \\
\hline Sequencial: 27 & Global: 30 \\
\hline
\end{tabular}

Tabela 1. Frequências e Interseção dos OA nos respectivos EA e dimensões.

Ao buscar respostas para a terceira pergunta de pesquisa: pode-se falar em correlação/mapeamento entre EA e OA?, foi possível perceber uma interseção dos OA, presentes em EA de uma mesma dimensão. Na Tabela 2 destacamos estas interseções. Na dicotomia Processamento, que inclui os estilos Ativo e Reflexivo, 22 recursos educativos atendem aos dois perfis. Isso indica que do total de OA recuperados para o estilo Ativo, $79 \%$ deles, também são associados ao Estilo Reflexivo. Este índice também é significativo no Estilo Reflexivo, em que $69 \%$ dos OA também estão relacionados ao estilo antagônico de sua dimensão. Na dicotomia Entrada, 84\% dos recursos digitais do Estilo Visual são associados ao Estilo Verbal, este último apresenta 63\% de OA comuns. A interseção do Estilo Visual é alarmante, pois apenas $16 \%$ dos recursos educativos são específicos a este perfil. Consequentemente, predizer o EA de um aluno com base no OA acessado seria um método ineficaz já que apresentaria diversas inconsistências.

Os Estilos Sensitivo e Intuitivo da dimensão Percepção também apresentaram intersecções consideráveis, 63\% dos OA indicados aos alunos sensitivos são similares na dimensão oposta. A interseção no Estilo Intuitivo atinge o valor de $67 \%$, significando que mais da metade dos recursos educativos indicados a seu perfil estão sendo associados, também, ao Estilo Sensitivo. Não obstante, nos estilos Sequencial e Global, o mesmo fato foi observado, sendo que no Estilo Sequencial a interseção alcançada foi igual a $67 \%$ e no Estilo Global referente a $60 \%$.

\footnotetext{
${ }^{3}$ Os recursos educativos associados a cada perfil podem ser encontrados em: https://drive.google.com/file/d/1FHEZrpKjL-XcEmSJoQmdftzPAOtXNMp9/view?usp=sharing
} 
IX Congresso Brasileiro de Informática na Educação (CBIE 2020)

Anais do XXXI Simpósio Brasileiro de Informática na Educação (SBIE 2020)

\begin{tabular}{|c|c|c|}
\hline Dimensão & Estilos & $\begin{array}{c}\text { Valor da } \\
\text { Interseção }\end{array}$ \\
\hline Entrada & Visual e Verbal & 21 \\
\hline Percepção & Sensitivo e Intuitivo & 20 \\
\hline Processamento & Ativo e Reflexivo & 22 \\
\hline Organização & Sequencial e Global & 18 \\
\hline
\end{tabular}

Tabela 2. Interseções dos Objetos de Aprendizagem presentes em uma mesma
dimensão do modelo de Estilo de Aprendizagem

Segundo as interseções referentes a cada Estilo de Aprendizado é possível perceber que, em todos os casos, mais da metade dos Objetos de Aprendizagem são indicados à perfis diferentes de uma mesma dimensão. Partindo do conceito de Estilo de Aprendizagem, é cognoscível que ele configure as características de aprendizagem individuais dos alunos. Ao realizar a associação entre os EA e o tipos de OA, espera-se que o recurso educativo indicado esteja em conformidade com as características de aprendizagem ditadas em um EA específico. Se estes recursos não apresentam uma unicidade a cada perfil, pode-se afirmar que esta associação apresenta inconsistências no processo de recomendação de OA. Assim, o seguinte questionamento é colocado em pauta: até que ponto o OA é classificador do perfil do aluno para auxiliar na predição do EA? Ou o contrário, até que ponto o EA é classificador do perfil do aluno para recomendação de $\mathrm{OA}$ ?

$\mathrm{O}$ fato de um mesmo recurso educativo atender a Estilos de Aprendizagem de uma mesma dimensão anula a eficácia da predição, uma vez que, dos 46 OA recuperados nesta revisão, apenas $22 \%$ deste total, são únicos a determinados EA. Dessa forma, garantir a predição dos EA, tendo como base, por si só, o acesso dos recursos de aprendizagem não garante uma identificação eficaz. Sabemos que a adaptação de um Sistema de Tutoria Inteligente vai além das recomendações e apresentações dos Objetos de Aprendizagem. Existem outros fatores utilizados para entender o comportamento do aluno e propiciar uma personalização do ambiente mais efetiva, como podemos encontrar no trabalho de [Yannibelli et al. 2006]. No entanto, é inegável a importância do Estilo de Aprendizagem e Objeto de Aprendizagem neste processo. Assim, este artigo apresenta resultados que levantam questionamentos e análises importantes sobre a temática em questão, baseados na análise da literatura, ainda não explorados nesta magnitude.

As interseções entre as dicotomias de estilos trazem à tona problemas em aberto na literatura como a efetividade dos EA em representar as preferências individuais dos alunos. Essas inconsistências na forma como os recursos educativos são indicados a determinados perfis de alunos corroboram com os trabalhos de [Rohrer and Pashler 2012], [Curry 1990], [Coffield 2004], suscitando questionamentos acerca da efetividade dos Estilos de Aprendizagem no processo de recomendação de Objetos de Aprendizagem.

\section{Considerações Finais}

O propósito desta pesquisa foi entender a relação entre o Objeto de Aprendizagem e Estilo de Aprendizagem tendo como objeto de estudo um conjunto de publicações recuperadas através da execução da Revisão Sistemática da Literatura. Ao construir o mapeamento 
entre o OA e EA, calculou-se a interseção da ocorrência de recursos educativos presentes em uma mesma dimensão. Os valores obtidos, ou seja, intersecções com valores acima de $60 \%$, demonstram a inviabilidade de utilizar somente os modelos de Estilos de Aprendizagem na predição ou personalização de conteúdo. Por este motivo, em trabalhos futuros sugere-se o estudo de aspectos, tais como: traços de personalidade e padrão de comportamento em ambientes educacionais como potenciais fatores de predição. Também a realização de estudo aprofundado entre o OA e EA e uma possível ponderação da relevância do OA para cada EA.

As limitações observadas na execução desta RSL são comuns àquelas apontadas por diversos autores na literatura, [Valaski et al. 2011], [do Nascimento et al. 2017]: possíveis decisões subjetivas durante o processo de eliminação dos artigos, tendo em vista a escassez de informações nas publicações.

\section{Referências}

Behar, P. A., Passerino, L. M., and Bernardi, M. (2007). Modelos pedagógicos para educação a distância: pressupostos teóricos para a construção de objetos de aprendizagem. RENOTE: revista novas tecnologias na educação [recurso eletrônico]. Porto Alegre, $R S$.

Carvalho, V. (2015). Uma abordagem para recuperação e recomendação automática, dinâmica e não determinística de objetos de aprendizagem baseada em estilos de aprendizagem, em metadados no padrão ieee lom e em elementos de web semântica. In Anais dos Workshops do Congresso Brasileiro de Informática na Educação, volume 4, page 135 .

Coffield, F. e. a. (2004). Learning styles and pedagogy in post-16 learning. a systematic and critical review. newcatle university report on learning styles. Cited: $1031,2011$. http://www.Isda.org.uk/files/PDF/1543.pdf.

Curry, L. (1990). A critique of the research on learning styles. Educational leadership, 48(2):50-56.

do Nascimento, P., Barreto, R., Primo, T., Gusmão, T., and Oliveira, E. (2017). Recomendação de objetos de aprendizagem baseada em modelos de estilos de aprendizagem: Uma revisão sistemática da literatura. In Brazilian Symposium on Computers in Education (Simpósio Brasileiro de Informática na Educação-SBIE), volume 28, page 213.

Dorça, F. A., Lima, L. V., Fernandes, M. A., and Lopes, C. R. (2011). Detecção e correção automática de estilos de aprendizagem em sistemas adaptativos para educação. Revista de Informática Teórica e Aplicada, 18(2):178-204.

Dorça, F. A., Silva, D. H., Lima, L. V., Fernandes, M. A., and Lopes, C. R. (2013). Uma abordagem para geração automática de conteúdo personalizado através da recomendação estocástica de objetos de aprendizagem no processo de ensino em sistemas adaptativos e inteligentes para educação. In Brazilian Symposium on Computers in Education (Simpósio Brasileiro de Informática na Educação-SBIE), volume 24, page 838.

Felder, R. M., Silverman, L. K., et al. (1988). Learning and teaching styles in engineering education. Engineering education, 78(7):674-681. 
IX Congresso Brasileiro de Informática na Educação (CBIE 2020)

Anais do XXXI Simpósio Brasileiro de Informática na Educação (SBIE 2020)

Franzoni, A. L., Assar, S., Defude, B., and Rojas, J. (2008). Student learning styles adaptation method based on teaching strategies and electronic media. In 2008 Eighth IEEE International Conference on Advanced Learning Technologies, pages 778-782. IEEE.

Graf, S. et al. (2009). Advanced adaptivity in learning management systems by considering learning styles. In Proceedings of the 2009 IEEE/WIC/ACM International Joint Conference on Web Intelligence and Intelligent Agent Technology-Volume 03, pages 235-238. IEEE Computer Society.

Kitchenham, B. (2004). Procedures for performing systematic reviews. Keele, UK, Keele University, 33(2004):1-26.

Kolekar, S. V., Pai, R. M., and MM, M. P. (2017). Prediction of learner's profile based on learning styles in adaptive e-learning system. International Journal of Emerging Technologies in Learning (iJET), 12(06):31-51.

Li, M., Ogata, H., Hou, B., Uosaki, N., and Yano, Y. (2012). Personalization in contextaware ubiquitous learning-log system. In 2012 IEEE Seventh International Conference on Wireless, Mobile and Ubiquitous Technology in Education, pages 41-48. IEEE.

Neiva, F. W. and da Silva, R. L. d. S. (2016). Revisão sistemática da literatura em ciência da computação um guia prático. Technical report, Universidade Federal de Juiz de Fora.

Peña, C. I., Marzo, J. L., De la Rosa, J. L., and Fabregat, R. (2002). Un sistema de tutoría inteligente adaptativo considerando estilos de aprendizaje. Revista UIS ingenierías, $1(2): 17-29$.

Rohrer, D. and Pashler, H. (2012). Learning styles: Where's the evidence?. Online Submission, 46(7):634-635.

Silva, S. B., Machado, V. P., and Araújo, F. N. (2014). Sistema tutor inteligente baseado em agentes na plataforma moodle para apoio as atividades pedagógicas da universidade aberta do piauí. In Anais dos Workshops do Congresso Brasileiro de Informática na Educação, volume 3, page 592.

Valaski, J., Malucelli, A., and Reinehr, S. (2011). Revisão dos modelos de estilos de aprendizagem aplicados à adaptação e personalização dos materiais de aprendizagem. In Brazilian Symposium on Computers in Education (Simpósio Brasileiro de Informática na Educação-SBIE), volume 1.

Wenger, E. (2014). Artificial intelligence and tutoring systems: computational and cognitive approaches to the communication of knowledge. Morgan Kaufmann.

Yannibelli, V., Godoy, D., and Amandi, A. (2006). A genetic algorithm approach to recognise students' learning styles. Interactive Learning Environments, 14(1):55 - 78.

Zaina, L. (2010). Uma abordagem para recomendação de objetos de aprendizagem em ambientes educacionais. Revista de Computação e Tecnologia (ReCeT). ISSN 21767998, 2(1):23-32. 